\title{
\$FMH Flash
}

A V R I L 2011

\section{Brèves}

\section{La santé pour tous}

Barbara Weil est au service du département Promotion de la santé et prévention de la FMH depuis 19 ans; elle le dirige depuis 1996. Représentant la FMH au sein de nombreuses commissions, elle a des journées de travail particulièrement animées. Cette collaboration avec les autres organisations est importante car elle permet aux différents acteurs d'unir leurs forces.

Barbara Weil, pourquoi la FMH s'engage-t-elle dans la promotion de la santé et la prévention?

La FMH a des objectifs statutaires clairs: «Le médecin a pour mission de protéger la vie de l'être humain, de promouvoir et de maintenir sa santé...»; elle a par ailleurs défini dans sa stratégie que le corps médical doit faire entendre sa voix: «Pas de politique de santé sans la FMH.» Il est important de mettre à profit le savoir des médecins déjà lors de la conception de stratégies et de campagnes en matière de prévention, de façon à ce que rien ne soit planifié sans tenir compte des besoins réels de la population ou des fournisseurs de prestations. Enfin et surtout,

Barbara Weil: «Sans coopération, pas de promotion de la santé ni de prévention.» riques pour ses décisions portant sur des projets de politique de santé. Nous nous concentrons sur différents thèmes comme les maladies transmissibles, les maladies chroniques non transmissibles, la dépendance et les maladies psychiques. Outre le soutien logistique à la Commission Prévention de la FMH, nous participons à des commissions nationales, notamment au groupe d'accompagnement du Programme national alcool, à l'Association pour la prévention du tabagisme, à la Coordination politique des addictions et à l'Alliance Compétences en matière de santé; nous sommes également actifs au sein du groupe spécialisé «Mental Health» de Santé Publique Suisse et représentons la FMH dans diverses commissions fédérales.

A quoi ressemble une journée de travail typique? C'est très varié et cela peut parfois être très mouvementé. Je commence par exemple par lire des documents et des projets de loi en vue d'une prise de position. L'après-midi, je me rends à une séance de l'Alliance Compétences en matière de santé, et le jour suivant je participe à l'assemblée des délégués de l'Association pour la prévention du tabagisme. En plus de l'examen des différents documents de séance, je rassemble des données à l'intention des médias, j'intègre les remarques des sociétés de discipline médicale dans la prise de position en cours, et je prépare une conférence. Je peux pour cela compter sur le soutien énergique de Rosmarie Logovi. Nous rencontrons également régulièrement la Dr $\mathrm{C}$. Romann, membre du Comité central de la FMH et responsable du domaine Promotion de la santé et prévention, lors de nos séances internes.

Quelle importance revêt la collaboration avec les autres organisations?

Dans le domaine Promotion de la santé et prévention / Santé publique, rien ne peut être entrepris sans coopérer en matière de stratégies 
et de mesures. La Suisse est trop petite pour appliquer 26 programmes différents de prévention du tabac, 35 stratégies régionales en faveur d'un équilibre pondéral sain et j'en passe. Je suis naturellement d'avis que les spécificités régionales doivent être prises en compte - mais il ne faut pas non plus chaque fois réinventer la roue.

Et vous, comment entretenez-vous votre santé? Avec un peu de sport, du jardinage, et des concerts.

\section{Départements}

\section{Services}

\section{Economie d'électricité: nous y contribuons}

La réception de cette lettre nous a particulièrement réjoui: Energie Wasser Bern (ewb), notre fournisseur d'électricité, nous crédite un «Bonus pour économie de courant» pour nous remercier d'avoir économisé 2,6\% de courant en 2010. Voici comment nous y sommes parvenus: nous avons commencé par analyser la consommation d'énergie du Secrétariat général de la FMH. A l'aide d'un conseiller d'ewb, nous avons recherché les appareils gourmands en énergie et défini des mesures pour améliorer la situation. Nous en avons déjà mises en œuvre quelquesunes - avec succès, comme nous avons pu le constater. L'économie de courant réalisée vient principalement de notre passage à la gestion virtuelle de nos serveurs informatiques: à la place de 20 serveurs physiques, nos applications fonctionnent maintenant sur seulement six serveurs! Mais de nombreux petits gestes permettent également d'économiser du courant: éteindre complètement les ordinateurs, les imprimantes et les écrans avant de rentrer chez soi, éteindre la lumière dès qu'elle n'est plus nécessaire, utiliser si possible des ampoules halogènes économiques, installer un éclairage de plafond économique au lieu de lampes de bureau halogènes gourmandes en énergie, désactiver plus rapidement la lumière extérieure et celle du garage. Bref, nous avons fait un premier pas en avant, mais il reste encore beaucoup à faire: continuons sur cette voie!

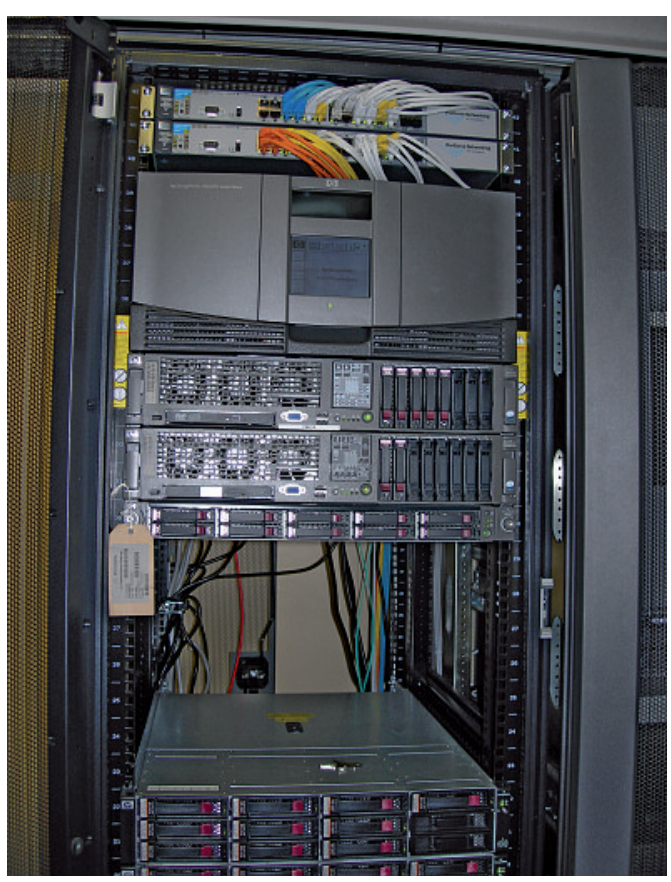

Diminution de la consommation d'énergie: la FMH est passée d'une gestion physique à une gestion virtuelle de ses 20 serveurs

\section{Personnel}

\section{Nouvelle année - nouveaux visages}

Nous souhaitons la bienvenue à Carla Trachsel, qui renforce depuis janvier l'équipe du Secrétariat de direction. Carla Trachsel est notamment responsable de l'administration du Forum du sauvetage. Nous souhaitons également la bienvenue à Isabelle Bertholet, qui travaille depuis janvier au sein du Service de traduction et qui remplace Catherine Schläfli, qui a pris un congé non-payé de six mois pour se consacrer à un projet artistique dans le cadre d'une bourse d'études. Isabelle Bertholet sera également la remplaçante d'Antoinette Mazouer, qui prendra sa retraite à la fin du mois de mai.

\section{Communication}

\section{Rencontre avec les parlementaires: mieux vaut prévenir que guérir}

«La loi sur la prévention: des perspectives nationales pour la promotion de la santé et la prévention», tel a été le thème de la $12^{\mathrm{e}}$ rencontre de la FMH avec les parlementaires. Les nombreux invités du monde politique, 
des pouvoirs publics, des médias et de la santé publique ont découvert les enjeux de la nouvelle Loi sur la prévention. Pascal Strupler, directeur de l'Office fédéral de la santé publique, Thomas Mattig, directeur de la Fondation suisse pour la promotion de la santé, et Ignazio Cassis, viceprésident de la FMH et conseiller natio-

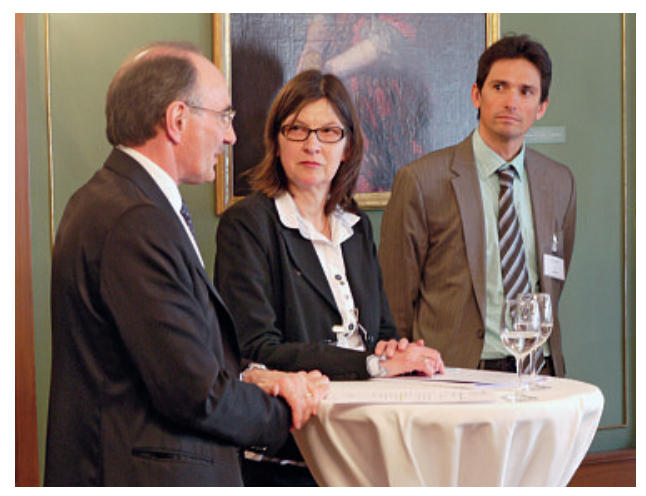

Les invités à la table ronde lors de la rencontre parlementaire de la FMH: Felix Gutzwiller, conseiller aux Etats, Silvia Schenker, conseillère nationale, et Thomas Mattig, directeur de la Fondation suisse pour la promotion de la santé...

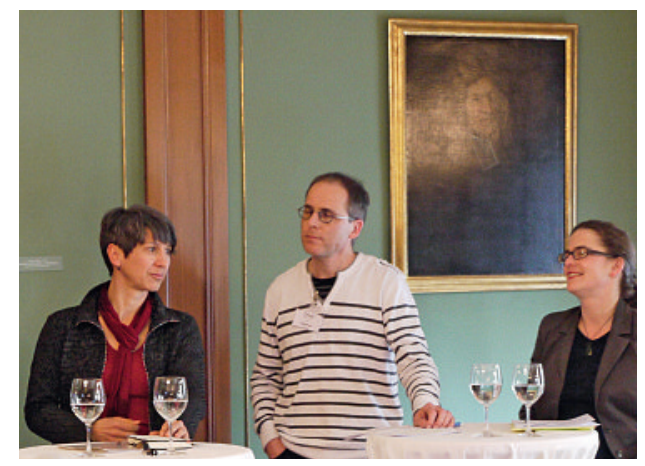

... et Anna Sax, animatrice, Alex Reichmuth, rédacteur à la Weltwoche, et Salome von Greyerz, de l'Office fédéral de la santé publique.

nal PLR, ont défendu la valorisation, sur les plans juridique et organisationnel, de la promotion de la santé et de la prévention. Au cours de la discussion plénière, Alex Reichmuth, rédacteur à la Weltwoche, a estimé que la Loi sur la prévention mettait les gens sous tutelle, ce que Silvia Schenker, conseillère nationale PS, et Felix Gutzwiller, conseiller aux Etats PLR, n'ont pas manqué de contredire.

Cette discussion animée a retenu toute l'attention du public. Une fois de plus, la FMH a réussi à apporter une contribution intéressante à un dossier politique important et vivement débattu. Cette question sera très vraisemblablement portée à l'ordre du jour de la session d'été.

\section{Who is who?}

Avez-vous une question concernant votre formation postgraduée? Une demande dans le domaine des tarifs? Ou aimeriez-vous commander le prospectus de la statistique médicale 2010?

Nous vous aidons volontiers en vous indiquant à qui vous adresser.

La formation postgraduée et continue: Institut suisse pour la formation médicale postgraduée et continue (ISFM), 03135911 11, siwf@fmh.ch L'affiliation à la FMH, la HPC: administration des membres, $031359 \quad 11 \quad 11$, dlm@fmh.ch

Les tarifs, SwissDRG: service tarifaire, 06228796 96, tarife@fmh.ch, swissdrg@fmh.ch Le droit, les assistantes médicales: service juridique, 03135911 11, lex@fmh.ch, mpa@fmh.ch

La statistique médicale, ReMed: département Données, démographie et qualité (DDQ), 03135911 11,ddq@fmh.ch

Le Bulletin des médecins suisses: communication, 03135911 50,kommunikation@fmh.ch

\section{Tarifs}

\section{TARVISION: révision TARMED}

A fin 2010, le domaine Tarifs et Conventions de la FMH a informé les sociétés de discipline concernées du déroulement prévu pour le projet TARVISION. Depuis lors, ces sociétés et la FMH travaillent de manière intensive à la révision de la structure tarifaire TARMED. Durant la première phase du projet, les sociétés de discipline se penchent sur la révision des paramètres les plus importants de leurs positions tarifaires les plus fréquentes. Le domaine Tarifs et Conventions leur offre un appui individuel et s'emploie simultanément 
- dans le cadre de la deuxième phase du projet - à actualiser les principales valeurs des modèles de coûts.

L'objectif de cette révision est d'adapter la structure tarifaire TARMED aux conditions actuelles, indépendamment des transferts de coûts qui pourront en résulter, afin de pouvoir montrer au final la réalité des coûts.

\section{SwissDRG: aide à la documentation}

Dans le cadre de leur profession, les médecins réunissent déjà des informations pour les échanges avec leurs collègues, à des fins statistiques, ou pour se prémunir contre une action juridique. L'introduction des forfaits par cas génère une exigence supplémentaire, celle de documenter les éléments à la base de la facturation. Car seules des prestations documentées peuvent être codifiées et donc rémunérées.

C'est également valable pour les codes complexes figurant dans la classification des procédures CHOP 2011. Ces codes contiennent des exigences minimales telles que le traitement par des spécialistes de plusieurs disciplines, l'échelonnement d'après la durée et les unités thérapeutiques, etc. Lorsque ces exigences minimales ne sont pas remplies, le code complexe ne peut pas être utilisé.

Pour relier les prestations individuelles sous une forme adéquate et compréhensible, la FMH a, sur demande des sociétés de discipline, élaboré des modèles de documentation. Ces modèles sont à la disposition des membres de la FMH sous $w w w . f m h . c h \rightarrow$ Tarifs $\rightarrow$ SwissDRG $\rightarrow$ Documentation médicale des codes CHOP complexes. Bien entendu, il s'agit ici de propositions qui peuvent être utilisées et adaptées de manière individuelle.

Cette année aussi, la FMH accorde son soutien aux sociétés de discipline et à leurs experts pour le dépôt de propositions visant à améliorer le système SwissDRG et les classifications. Cette procédure commencera le 5 mai 2011; le délai d'envoi est fixé au 5 juillet 2011 pour les propositions concernant le développement du catalogue des forfaits par cas et au 20 septembre 2011 pour les propositions CHOP et CIM-10. Le 16 mai 2011, la FMH organisera une séance d'information pour les sociétés de discipline et les organisations faîtières.

\section{La statistique médicale} de la FMH

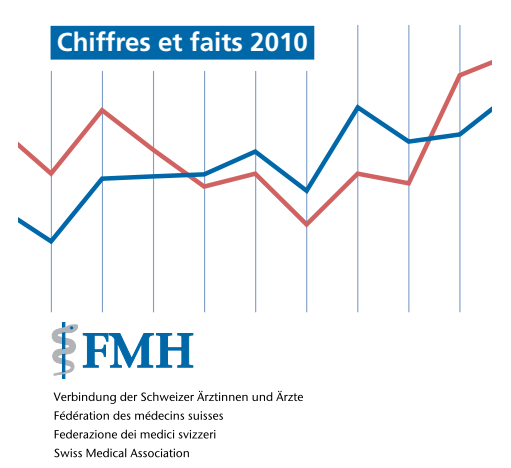

Un papillon compact et clair pour la statistique médicale suisse 2010

\section{Données, démographie, qualité}

Interactivité pour la statistique médicale Les chiffres les plus récents de la démographie médicale suisse ont été publiés: voulez-vous savoir dans quels cantons les 30272 médecins répertoriés travaillent ou quel est le taux de femmes dans une discipline déterminée? Pas de problème, en quelques clics de souris, vous parvenez sur les informations souhaitées. Comme elles sont présentées de manière interactive, vous pouvez les obtenir sous forme de tableau ou de graphique, les imprimer et les exporter. Vous trouverez tout le détail de la statistique médicale FMH 2010 comme d'habitude sur notre site internet $w w w . f m h . c h$ $\rightarrow$ Autres thèmes $\rightarrow$ Démographie médicale. Le papillon, qui la présente sous la forme d'un aperçu compact et clair, a également été mis à jour. Le département Données, démographie et qualité se tient à votre entière disposition pour répondre à vos questions spécifiques et vous donner des compléments d'information. Pour toute question ou pour commander le prospectus, veuillez écrire à ddq@fmh.ch. 